\title{
ALK-positive anaplastic large cell lymphoma with prominent bone involvement in a 13-year-old boy
}

This article was published in the following Dove Press journal:

OncoTargets and Therapy

II January 2016

Number of times this article has been viewed

\section{Chen Tian \\ Yong Yu \\ Hongliang Yang \\ Lei Zhu \\ Yafei Wang \\ Yizhuo Zhang}

Key Laboratory of Cancer Prevention and Therapy, Tianjin Medical

University Cancer Institute and

Hospital, National Clinical Research

Center for Cancer, Tianjin, People's

Republic of China
Correspondence: Yizhuo Zhang

Key Laboratory of Cancer Prevention and Therapy, Tianjin Medical University Cancer Institute and Hospital, National Clinical Research Center for Cancer, Huanhuxi Road, Hexi District, Tianjin 300060, People's Republic of China Tel/fax +862223340123

Email yizhuozhangIII@I63.com
Introduction: Anaplastic lymphoma kinase-positive (ALK+) anaplastic large cell lymphoma (ALCL) is a type of non-Hodgkin lymphoma, which has strong expression of cluster of differentiation (CD)-30 and ALK. ALCL sometimes can involve the bone marrow, and in advanced stages, it can produce destructive bone lesions. But ALK+ ALCL with prominent bone involvement is very rare, especially in children.

Case report: A 13-year-old boy presented with waist pain and low-grade fever for 8 months. The biopsy of soft tissue lesions around the thoracic spine showed that these cells were positive for ALK-1, CD30, leukocyte common antigen, CD3, CD4, and CD8, as well as being negative for epithelial membrane antigen and pan-cytokeratin, which revealed ALCL. After six cycles of a regimen consisting of hyperfractionated cyclophosphamide, vincristine, doxorubicin, and dexamethasone/methotrexate and cytarabine (hyper-CVAD/MA) and autologous hematopoietic stem cell transplantation, he achieved complete remission (CR).

Conclusion: It is generally believed that the regimen consisting of cyclophosphamide, hydroxydaunorubicin (doxorubicin), vincristine, and prednisolone (CHOP) is also applicable to ALCL. Because of the tendency of rapid progression and the frequency of B symptoms, ALCL in children and young adults is treated with high-grade chemotherapy such as hyper-CVAD/MA.

Keywords: anaplastic large cell lymphoma, anaplastic lymphoma kinase, bone involvement, hyper-CVAD/MA

\section{Introduction}

Anaplastic large cell lymphoma (ALCL) is a distinct clinicopathologic entity of non-Hodgkin lymphoma (NHL), which comprises approximately $15 \%$ of all NHL cases in children. ALCL in children commonly presents with advanced systemic disease. ${ }^{1}$ Although ALCL frequently involves the bone marrow, primary or secondary involvement to bone is rare. Here, we report a case of anaplastic lymphoma kinase positive $(\mathrm{ALK}+)$ ALCL with prominent bone involvement in a 13-year-old boy.

\section{Case report}

A 13-year-old boy presenting with waist pain and low-grade fever for 8 months was examined in our hospital. Physical examination revealed lymphadenopathy in the neck and hepatosplenomegaly. Serum lactate dehydrogenase (LDH) was elevated to 600 IU/L (normal: 200-460 IU/L), but other laboratory data were normal. Computed tomography (CT) scan of chest and abdomen revealed hepatosplenomegaly. ${ }^{18}$ F-Fluorodeoxyglucose (FDG) positron emission tomography (PET) scanning showed increased FDG avidity involving the thoracic vertebrae 1 and 8, the left ribs 4 and 6, the thoracic spine, sacrum, bilateral ilium, left pubis, and femur, suggesting lymphoma involvement (Figure 1). PET-CT also showed an FDG-avid neck mass, suggesting lymphadenopathy. A CT-guided biopsy of soft tissue lesions around the thoracic 


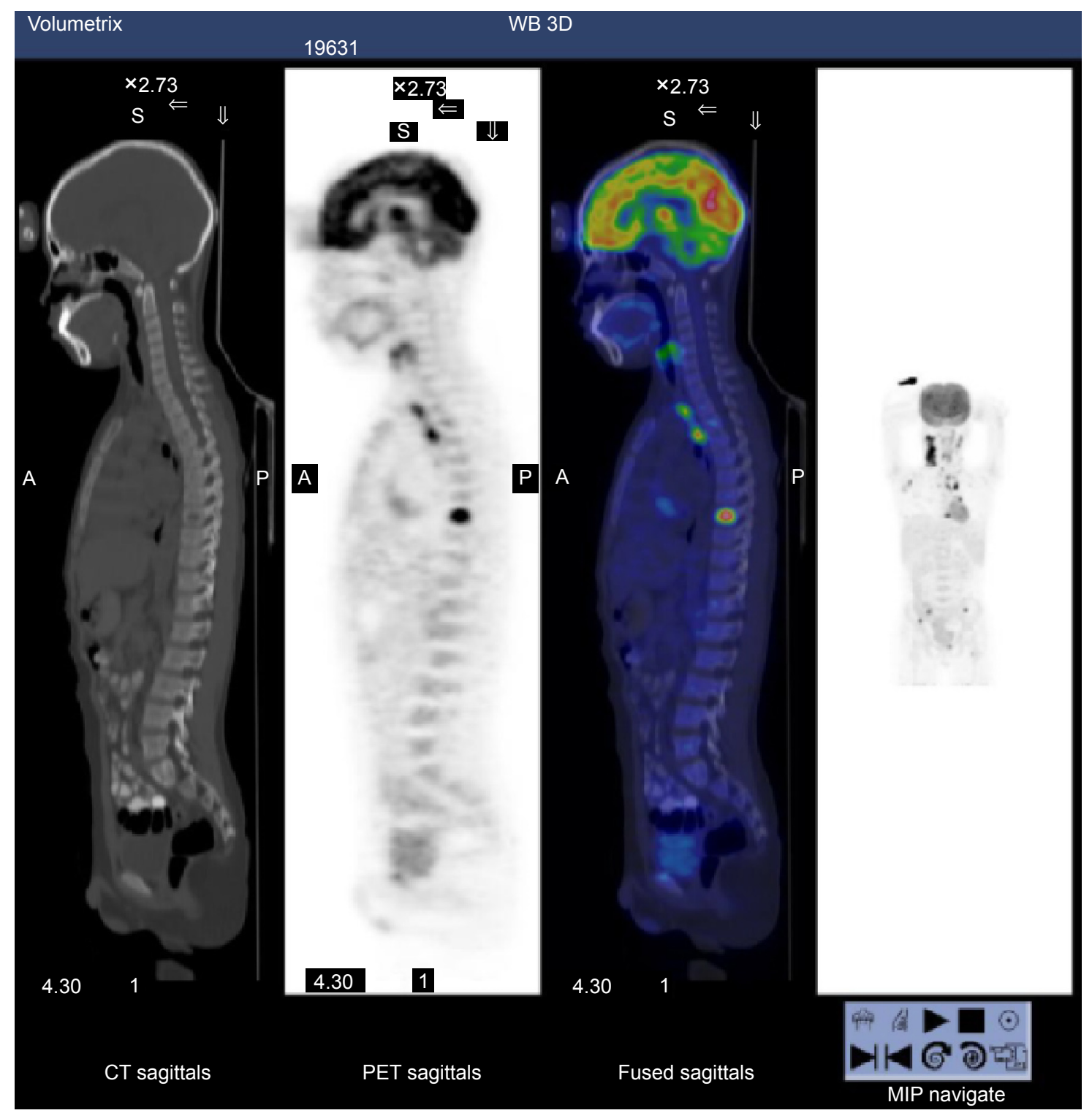

Figure I PET-CT of the patient.

Abbreviations: A, anterior; CT, computed tomography; PET, positron emission tomography; P, posterior; S, superior; MIP, multum in parvo.

spine revealed ALCL. These cells were positive for ALK-1 (Figure 2A), CD30 (Figure 2B), leukocyte common antigen, CD3, CD4, and CD8, as well as being negative for epithelial membrane antigen and pan-cytokeratin. Bone marrow aspiration and trephine biopsy showed no infiltration. He was diagnosed as having ALK-positive ALCL with prominent bone involvement. After two cycles of chemotherapy with hyperfractionated cyclophosphamide, vincristine, doxorubicin, and dexamethasone/methotrexate and cytarabine (hyperCVAD/MA), his condition improved, and the level of serum LDH returned to normal. After four cycles of hyper-CVAD/ MA chemotherapy, PET-CT showed no significant uptake of FDG. After six cycles of hyper-CVAD/MA regimen, PET-CT showed no uptake of FDG, suggesting complete remission (CR). Then, he underwent autologous hematopoietic stem cell transplantation (AHSCT) as consolidation therapy. At present, he has remained in the CR stage for 2 years. Maintenance chemotherapy has not been given.

\section{Discussion}

First described in 1985 by Stein et al, ${ }^{1}$ ALCL is characterized by CD30 expression on the malignant cells. Overexpression of the ALK protein is believed to be the main molecular driver of oncogenesis in the overwhelming majority of pediatric ALCL cases. ${ }^{2}$ The World Health Organization divides ALCL into three separate entities based on this distinct molecular pathway: ALK-positive ALCL, ALK-negative ALCL, and primary cutaneous ALCL. Dissimilar from ALCL in adults, 
A

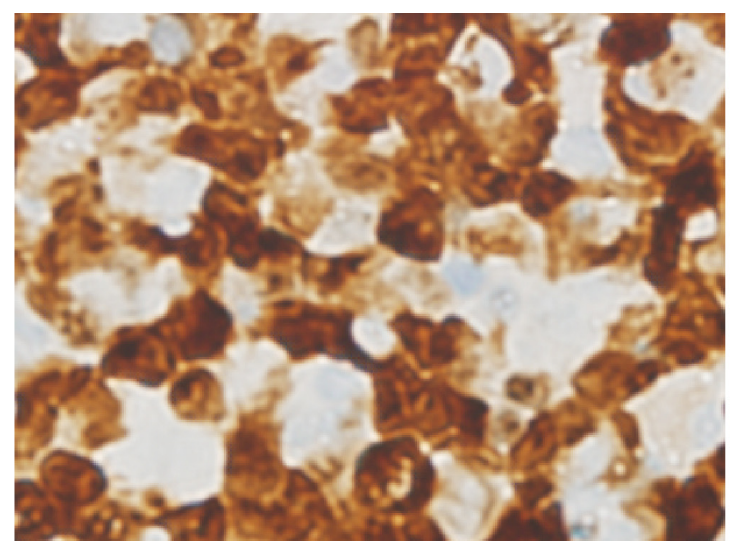

B

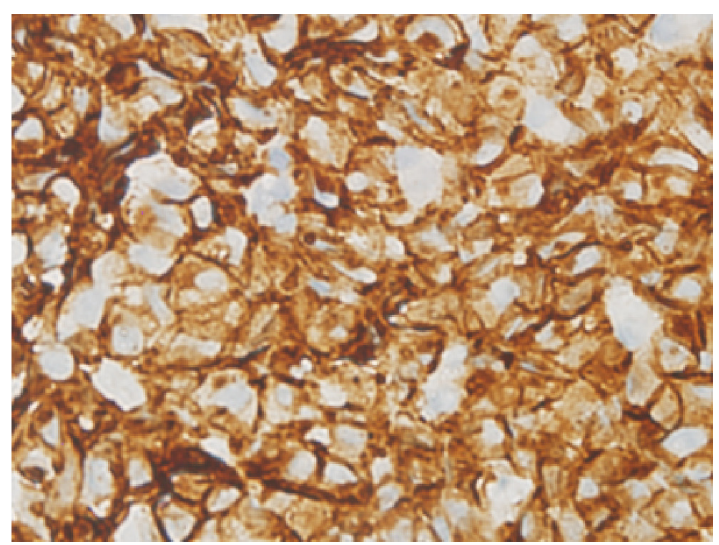

Figure 2 Biopsy of soft tissue lesions.

Notes: (A) ALK staining, original magnification: $\times 200$; (B) CD30 staining, original magnification: $\times 200$.

Abbreviations: ALK, anaplastic lymphoma kinase; $C D$, cluster of differentiation.

ALCL in children is nearly universally ALK positive. ${ }^{3}$ The median age at diagnosis for children with ALCL is approximately 12 years. Pediatric ALCL is usually characterized by advanced disease at presentation, with a high incidence of extranodal involvement. ${ }^{4}$ Although ALCL frequently involves the bone marrow, it rarely produces localized bone lesions. There are few reports in the literature of primary bone ALCL in children. ${ }^{5}$ The case we report here was of ALCL with prominent bony involvement. The diagnosis of primary bony ALCL is based on morphology and immunology. The case we report here was diagnosed with PET-CT and pathology.

Varying strategies for treating children with ALCL have emerged over the past 2 decades, but these display similar treatment efficacy. Some clinical trials have used chemotherapy such as cyclophosphamide, hydroxydaunorubicin (doxorubicin), vincristine, and prednisolone (CHOP-type) regimens. In addition, it is generally believed that the $\mathrm{CHOP}$ regimen is applicable to ALCL. Sun et $\mathrm{al}^{6}$ reported that a risk-stratified treatment with a modified B-NHL-BFM-90 protocol (cyclophosphamide, ifosfamide, vincristine, etoposide, adriamycin, high-dose [HD]-methotrexate, vindesine, dexamethasone, cytarabine/HD-cytarabine) was efficacious for Chinese children with ALCL. It is reported that both autologous and allogeneic HSCT can offer the prospect of durable disease-free survival for ALCL cases in childhood and adolescence. Patients with $\mathrm{CR}$ at the time of AHSCT had significantly greater effect-free survival than patients with non-CR at the time of autologous HSCT. ${ }^{6}$ Consolidation therapy with AHSCT is a highly promising option only in patients with ALK-positive ALCL, with a CR at the time of transplantation and lack of extranodal disease being important predictors for a favorable outcome.

Recently, novel agents for the treatment of ALCL have emerged, such as brentuximab vedotin, a CD30-targeted antibody, which is now used for relapsed ALCL in clinical trials. ${ }^{7}$ Novel therapies may soon radically change the treatment paradigm for this disease and hopefully lead to less toxicity and improved outcomes. ${ }^{8}$ In this case, because of the tendency to rapid progression and the frequency of B symptoms, the patient was treated with high-grade chemotherapy such as hyper-CVAD/MA and he achieved CR, which was then followed by consolidation therapy with AHSCT.

\section{Acknowledgments}

This work was supported by grants 31301161,81570201 , and 81270603 from the National Natural Science Foundation of China (NSFC) and grant 13JCYBJC22800 from the Tianjin Natural Science Foundation.

The subject and his parents/guardians gave their informed written consent, and the study protocol was approved by the Ethics Committee of Tianjin Medical University Cancer Institute and Hospital.

\section{Disclosure}

The authors report no conflicts of interest in this work.

\section{References}

1. Stein H, Mason DY, Gerdes J, et al. The expression of the Hodgkin's disease associated antigen $\mathrm{Ki}-1$ in reactive and neoplastic lymphoid tissue: evidence that Reed-Sternberg cells and histiocytic malignancies are derived from activated lymphoid cells. Blood. 1985;66(4): $848-858$.

2. Wu C, Molavi O, Zhang H, et al. STAT1 is phosphorylated and downregulated by the oncogenic tyrosine kinase NPM-ALK in ALK-positive anaplastic large cell lymphoma. Blood. 2015;126(3):336-345.

3. Lovisa F, Cozza G, Cristiani A, et al. ALK kinase domain mutations in primary anaplastic large cell lymphoma: consequences on NPM-ALK activity and sensitivity to tyrosine kinase inhibitors. PLoS One. 2015; 10(4): 0121378.

4. Xing X, Feldman AL. Anaplastic large cell lymphomas: ALK positive, ALK negative, and primary cutaneous. Adv Anat Pathol. 2015;22(1):29-49.

5. Tian C, Wang Y, Zhang Y. ALK-positive anaplastic large cell lymphoma with prominent bone involvement. Br J Haematol. 2015;170(4):443. 
6. Sun X, Zhen Z, Lin S, et al. Treatment outcome of Chinese children with anaplastic large cell lymphoma by using a modified B-NHL-BFM-90 protocol. Pediatr Hematol Oncol. 2014;31:518-527.

7. Fukano R, Mori T, Kobayashi R, et al. Haematopoietic stem cell transplantation for relapsed or refractory anaplastic large cell lymphoma: a study of children and adolescents in Japan. Br J Haematol. 2015;168: $557-563$
8. Lowe EJ, Gross TG. Anaplastic large cell lymphoma in children and adolescents. Pediatr Hematol Oncol. 2013;30:509-519.

\section{Publish your work in this journal}

OncoTargets and Therapy is an international, peer-reviewed, open access journal focusing on the pathological basis of all cancers, potential targets for therapy and treatment protocols employed to improve the management of cancer patients. The journal also focuses on the impact of management programs and new therapeutic agents and protocols on

\section{Dovepress}

patient perspectives such as quality of life, adherence and satisfaction. The manuscript management system is completely online and includes a very quick and fair peer-review system, which is all easy to use. Visit http://www.dovepress.com/testimonials.php to read real quotes from published authors.

Submit your manuscript here: http://www.dovepress.com/oncotargets-and-therapy-journal 